\title{
Perfil epidemiológico e clínico dos pacientes submetidos a artroplastia total do joelho*
}

\section{Epidemiological and Clinical Profile of Patients Undergoing Total Knee Arthroplasty}

\author{
Fabrício Bolpato Loures ${ }^{1,2}$ Rogério Franco de Araújo Góes ${ }^{10}$ Caio Veloso Gusmão ${ }^{10}$ \\ Rodrigo Sattamini Pires e Albuquerque ${ }^{30}$ Pedro José Labronici ${ }^{30}$ \\ ${ }^{1}$ Hospital Santa Teresa, Petrópolis, RJ, Brasil \\ 2 Universidade do Estado do Rio de Janeiro, Rio de Janeiro, RJ, Brasil \\ ${ }^{3}$ Departamento de Ortopedia e Traumatologia, Universidade Federal \\ Endereço para correspondência Fabrício Bolpato Loures, PhD, Rua Dr. \\ Nelson de Sá Earp, 111/901, Centro, Petrópolis, Rio de Janeiro, 25680- \\ 195, Brasil (e-mail: fbolpato@gmail.com).
}

Fluminense, Niterói, RJ, Brasil

Rev Bras Ortop 2022;57(2):223-229.

\section{Resumo}

Palavras-chave

- artroplastia

- joelho

- comorbidade

- epidemiologia

- complicações pósoperatórias
Objetivo Definir o perfil epidemiológico e as comorbidades associadas dos pacientes submetidos à artroplastia total do joelho (ATJ) em dois hospitais de referência.

Métodos Durante o estudo observacional transversal, foram avaliados 294 pacientes submetidos à ATJ em 2 hospitais de atenção terciária. Coletou-se, por entrevista direta, o diagnóstico das comorbidades auto-relatadas. Foram calculados o índice de comorbidades funcionais (do inglês Functional Comorbidity Index, $\mathrm{FCl}$ ) e o índice modificado de cinco fatores de fragilidade (do inglês 5-Factor Modified Frailty Index, $\mathrm{mFI}-5$ ). Avaliou-se a incidência das comorbidades e a relação destas com os índices calculados.

Resultados A maioria dos pacientes da amostra foram mulheres $(p=0,000)$ entre a sétima e oitava décadas de vida. A hipertensão arterial sistêmica foi a patologia mais comum, seguida de obesidade e diabetes mellitus. O ICF apresentou uma relação direta com sexo feminino $(p=0,038)$ e a obesidade $(p<0,001)$. O mFI-5 apresentou relação somente com a obesidade $(p=0,022)$, e demonstrou uma chance maior de complicações nesse grupo.

Conclusão Os pacientes submetidos à AT] são essencialmente portadores de comorbidades clínicas que podem influenciar negativamente os resultados funcionais e comprometer a segurança do procedimento. A identificação dos fatores de risco pode contribuir para a segurança e melhor seleção dos candidatos a ATJ.

Trabalho desenvolvido no grupo de Cirurgia do Joelho, Hospital Santa Teresa, Petrópolis, RJ, Brasil.

recebido

03 de Junho de 2020

aceito

02 de Outubro de 2020

Publicado on-line

Março 31, 2021
DOI https://doi.org/

10.1055/s-0041-1724080. ISSN 0102-3616. (c) 2021. Sociedade Brasileira de Ortopedia e Traumatologia. All rights reserved.

This is an open access article published by Thieme under the terms of the Creative Commons Attribution-NonDerivative-NonCommercial-License, permitting copying and reproduction so long as the original work is given appropriate credit. Contents may not be used for commercial purposes, or adapted, remixed, transformed or built upon. (https://creativecommons.org/ licenses/by-nc-nd/4.0/)

Thieme Revinter Publicações Ltda., Rua do Matoso 170, Rio de Janeiro, RJ, CEP 20270-135, Brazil 


\begin{abstract}

\section{Keywords}

- arthroplasty

- knee

- comorbidity

- epidemiology

- postoperative complications

Objective To define the epidemiological profile and the associated comorbidities of patients submitted to total knee arthroplasty (TKA) in two reference hospitals .

Materials and Methods During the present cross-sectional observational study, 294 patients submitted to TKA were evaluated in 2 tertiary care hospitals. The diagnosis of self-reported comorbidities was collected by direct interview. The functional Comorbidity Index ( $\mathrm{FCl}$ ) and the 5-Factor Modified Frailty Index ( $\mathrm{mFI}-5)$ were calculated. The incidence of comorbidities and their relationship with the calculated indexes were evaluated.

Results Most of the patients in the sample were women $(p=0.000)$ between the seventh and eighth decades of life. Systemic arterial hypertension was the most common pathology, followed by obesity and diabetes mellitus. The $\mathrm{FCl}$ presented a direct relationship with females $(p=0.038)$ and obesity $(p<0.001)$. The mFI-5 was only associated to obesity ( $p=0.022)$, demonstrating a higher chance of complications in this group.

Conclusion Patients undergoing TKA are essentially carriers of clinical comorbidities that can negatively influence functional results and compromise the safety of the procedure. The identification of risk factors can contribute to the safety and better selection of TKA candidates.
\end{abstract}

\section{Introdução}

A artroplastia total do joelho (ATJ) é a opção de tratamento para pacientes portadores de osteoartrite $(\mathrm{OA})$ severa, refratária ao tratamento conservador. Essa cirurgia é capaz de melhorar a dor, proporcionando o alinhamento do membro e o retorno da mobilidade. ${ }^{1}$ Em sua maioria, os candidatos a ATJ são pacientes idosos, com baixa demanda física, e portadores de patologias associadas. ${ }^{2}$

A ATJ é um dos procedimentos mais bem-sucedidos do último século. ${ }^{3}$ Mais de 13 mil procedimentos foram realizados no Brasil em 2019., ${ }^{4,5}$ Esse número deve crescer de forma exponencial, impulsionado pelo envelhecimento da população, pelo aumento da obesidade, ${ }^{6}$ e pela maior demanda da população idosa por qualidade de vida. ${ }^{7}$ Apesar de segura, a cirurgia é considerada de grande porte, e está sujeita a possíveis complicações. ${ }^{8}$ Uma parcela significativa dos pacientes permanece insatisfeita após a cirurgia. Por essas razões, um índice que possa predizer o resultado funcional e os possíveis candidatos com maior chance de complicação seria extremamente útil.

Comorbidades são definidas como doenças ou condições médicas sem relação de causalidade com o diagnóstico principal, que coexistem com a patologia de interesse. ${ }^{9}$ Já complicações são eventos adversos que ocorrem durante uma doença, não sendo parte dessa patologia, embora possam resultar dela. ${ }^{10}$ A grande maioria dos pacientes candidatos a ATJ tem doenças clínicas associadas que podem, de forma significativa, contribuir para um maior índice de complicações per e pósoperatórias. ${ }^{11} \mathrm{~A}$ fim de predizer o resultado funcional após a ATJ e a possível chance de complicação, respectivamente, dois questionários práticos e objetivos podem ser usados: o índice funcional de comorbidades (do inglês Functional Comorbidity Index, $\mathrm{FCI}),{ }^{10}$ e o índice modificado de cinco fatores de fragilidade (do inglês 5-Factor Modified Frailty, mFI-5). ${ }^{11}$
O Brasil apresenta um grande déficit de assistência para cirurgias de artroplastia, que são procedimentos complexos e de alto custo. ${ }^{7}$ Antecipar os possíveis fatores que levam a complicações e piores resultados funcionais pode contribuir para um maior controle dos gastos e melhor seleção dos pacientes. Para tanto, é fundamental conhecer o perfil do paciente submetido a ATJ e as incidências das patologias associadas, o que melhora a qualidade da assistência e evita resultados desfavoráveis.

O objetivo deste estudo foi definir o perfil epidemiológico dos pacientes submetidos a ATJ e suas comorbidades associadas em dois hospitais de referência para a realização desse procedimento.

\section{Materiais e Métodos}

Este estudo observacional transversal foi aprovado pelos Comitês de Ética em Pesquisa (CEP) das instituições. Todos os procedimentos foram realizados de acordo com os padrões éticos da declaração de Helsinki de 1964, com suas alterações posteriores ou padrões éticos comparáveis. Os pacientes foram recrutados em dois hospitais de alta complexidade (atendimento terciário), referências para o procedimento de ATJ. A amostra foi composta por pacientes de vários níveis socioeconômicos. A etnia foi diversa, como é característica da população brasileira. Embora a manifestação fenotípica de cor da pele tenha pouca relação com a ancestralidade, essa característica foi documentada por autodeclaração.

O termo de consentimento livre e esclarecido (TCLE) foi assinado por todos os pacientes antes da sua inclusão na pesquisa. A coleta de dados foi realizada entre agosto de 2012 e dezembro de 2016. No total, 294 pacientes foram prospectivamente avaliados, sendo 203 do sexo feminino e 91 do sexo masculino. Os dados sobre comorbidades foram 
Perfil epidemiológico e clínico dos pacientes submetidos a AT] Loures et al. 225

coletados por entrevista direta, com diagnóstico autodeclarado. Após a documentação, foram calculados o $\mathrm{FCI}^{10}$ e o mFI$5 .{ }^{11}$ Conforme definido no protocolo de avalição pré-operatória, os pacientes foram pesados e tiveram sua estatura medida usando apenas roupas íntimas e um avental. Após essas medidas, foram calculados os índices de massa corporal, e realizou-se a categorização, de acordo com os parâmetros descritos pela Organização Mundial da Saúde (OMS). 0 grau radiográfico da $\mathrm{OA}$ foi classificado pelo autor principal conforme os critérios da classificação de Ahlbäck modificada Keyes et al. ${ }^{12}$

A análise descritiva dos dados foi feita com base em gráficos e cálculo de estatísticas descritivas (proporções de interesse, mínimo, máximo, média, mediana, e desvio padrão). Na análise inferencial, foram feitos testes de significância estatística. Dada a não normalidade das distribuições das variáveis FCI e mFI-5, foi usada uma abordagem não paramétrica. A comparação de dois grupos independentes foi feita pelo teste de Mann-Whitney, e, para a comparação de mais de dois grupos independentes, foi usado o teste não paramétrico de Kruskall-Wallis. 0 nível de significância adotado foi de $5 \%$. A associação entre duas variáveis quantitativas ou ordinais foi investigada pelo coeficiente de correlação de ordem de Spearman. A significância dos coeficientes de correlação foi avaliada pelo teste do coeficiente de correlação. As análises foram feitas usando-se os programas Statistical Package for the Social Sciences, (SPSS, IBM Corp., Armonk, NY, EUA), versão 22.0, e Microsoft Excel (Microsoft Corp., Redmond, WA, EUA), versão 2011.

\section{Resultados}

Foram submetidos aATJ 294 pacientes que preencheram os critérios de inclusão e aceitaram participar da pesquisa, assinando o TCLE. A amostra foi composta de 203 (69\%) pacientes do sexo feminino e 91 (31\%) do sexo masculino, havendo diferença na distribuição em relação ao sexo $(p=0,000)$.

A idade dos pacientes variou entre 40 e 86 anos. A idade média foi de 69 anos, sendo 68,3 anos para o grupo masculino e 69,4 anos para o grupo feminino. Não houve diferença significativa de faixa etária entre os sexos $(p=0,239)$. As estatísticas de idade estão demonstradas na - Tabela 1.

Os pacientes da amostra, em sua maioria, se declararam brancos $(72,7 \%)$, seguidos por pardos $(15,9 \%)$ e pretos $(11,4 \%)$. 0 índice de massa corporal (IMC) variou de 17,3 $\mathrm{kg} / \mathrm{m}^{2}$ a $44,1 \mathrm{~kg} / \mathrm{m}^{2}$, com média de $30 \mathrm{~kg} / \mathrm{m}^{2}$, desvio padrão de $4,9 \mathrm{~kg} / \mathrm{m}^{2}$, e mediana de $29,7 \mathrm{~kg} / \mathrm{m}^{2}$, e distribuição normal em ambos os grupos. As mulheres foram significativamente mais pesadas do que os homens $(p=0,017)$, embora não tenha havido diferença significativa na proporção de obesos $(p=0,70)$. As análises do IMC na amostra global e dividido por grupos estão demonstradas na - Tabela 2.

As comorbidades foram analisadas por gênero e por sua distribuição na amostra total. Não houve diferença estatisticamente significativa entre os sexos na distribuição das patologias, exceto para artrite reumatoide, que foi mais frequente no grupo feminino $(p=0,021)$. As principais estatísticas da distribuição das patologias estão demonstradas na -Tabela 3.
Tabela 1 Estatísticas de idade

\begin{tabular}{|l|l|l|l|}
\hline Idade (anos) & Feminino & Masculino & Global \\
\hline Média & 69,4 & 68,3 & 69,0 \\
\hline Mediana & 70,0 & 68,0 & 69,0 \\
\hline Desvio padrão & 7,5 & 7,9 & 7,6 \\
\hline Mínimo & 40,0 & 43,0 & 40,0 \\
\hline Máximo & 86,0 & 82,0 & 86,0 \\
\hline Gama & 46,0 & 39,0 & 46,0 \\
\hline $\begin{array}{l}\text { Coeficiente } \\
\text { de variação }\end{array}$ & 0,11 & 0,12 & 0,11 \\
\hline Número de casos & 203 & 91 & 294 \\
\hline $\begin{array}{l}\text { Valor de } p \text { do teste } \\
\text { de Kolmogorov-Smirnov }\end{array}$ & 0,053 & 0,044 & 0,002 \\
\hline $\begin{array}{l}\text { Valor de } p \text { do teste } \\
\text { de Shapiro-Wilk }\end{array}$ & 0,032 & 0,054 & 0,0011 \\
\hline $\begin{array}{l}\text { Valor de } p \text { do teste } \\
\text { de Mann-Whitney }\end{array}$ & 0,293 & \multicolumn{3}{|l}{} \\
\hline
\end{tabular}

Tabela 2 Distribuição pelo índice de massa corporal

\begin{tabular}{|l|l|l|l|}
\hline \multirow{2}{*}{$\begin{array}{l}\text { Classificação } \\
\text { do Peso }\end{array}$} & Gênero & \multicolumn{3}{|l|}{} \\
\cline { 2 - 4 } & Feminino & Masculino & Global \\
\hline Baixo neso & $1(0,5 \%)$ & 0 & $1(0,3 \%)$ \\
\hline Peso normal & $30(15 \%)$ & $18(20,2 \%)$ & $48(16,6 \%)$ \\
\hline Sobrepeso & $65(32,5 \%)$ & $35(39,3 \%)$ & $100(34,6 \%)$ \\
\hline Obesidade I & $64(32,0 \%)$ & $29(32,6 \%)$ & $93(32,2 \%)$ \\
\hline Obesidade II & $32(16 \%)$ & $6(6,7 \%)$ & $38(13,1 \%)$ \\
\hline Obesidade III & $8(4,0 \%)$ & $1(1,1 \%)$ & $9(3,1 \%)$ \\
\hline
\end{tabular}

A - Figura 1 ilustra a distribuição das patologias mais comuns na amostra total e sua distribuição por sexo.

A maior parte da amostra se encontrava entre 60 e 70 anos, e as comorbidades também foram mais frequentes nessa faixa etária, embora não tenha havido diferença estatística no $\mathrm{FCI}(p=0,221)$ e no $\mathrm{mFI}-5(p=0,365)$ entre as faixas etárias. A distribuição das patologias por faixa etária está demonstrada na - Tabela 4.

O FCI apresentou uma relação direta com o sexo feminino $(p=0,038)$ e com o IMC $(p<0,001)$, não estando relacionado com a idade $(p=0,221)$, a cor da pele $(p=0,058)$ ou a classificação radiográfica da OA (Ahlbäck) $(p=0,420)$. A única variável que apresentou correlação significativa com o mFI-5 foi o IMC $(p=0,022)$, não estando relacionado ao sexo $(p=0,237)$, à idade $(p=0,365)$, à cor da pele $(p=0,251)$ ou à gravidade da $\mathrm{OA}(p=0,874)$.

\section{Discussão}

A ATJ é uma das cirurgias mais realizadas no mundo. Nos Estados Unidos, são realizadas cerca de 700 mil ATJs por ano, atingindo a média de 213,28 ATJs para cada 100 mil habitantes. ${ }^{7}$ No Brasil, foram realizadas 13.210 ATJs no em $2019,{ }^{4,5}$ representando 6,29 ATJ/100 mil habitantes. Além 
Tabela 3 Frequência relativa das comorbidades clínicas

\begin{tabular}{|l|l|l|l|l|}
\hline \multirow{2}{*}{ Comorbidades } & \multicolumn{2}{|l|}{ Incidência da comorbidade (\%) } & $\begin{array}{l}\text { Valor de } p \text { do teste que } \\
\text { compara as incidências nos } \\
\text { grupos feminino e masculino }\end{array}$ \\
\cline { 2 - 5 } & Feminino & Masculino & $0,125^{*}$ \\
\hline $\begin{array}{l}\text { Hipertensão } \\
\text { arterial sistêmica }\end{array}$ & 67,5 & 58,2 & 64,6 & $0,070^{*}$ \\
\hline Obesidade & 52,0 & 40,4 & 48,4 & $0,912^{*}$ \\
\hline Diabetes mellitus & 14,8 & 14,3 & 14,6 & $0,021^{* *}$ \\
\hline Artrite reumatoide & 5,9 & 0,0 & 4,1 & $0,512^{* *}$ \\
\hline Dislipidemia & 4,4 & 2,2 & 3,7 & $1,000^{* *}$ \\
\hline Cardiopatia & 3,0 & 3,3 & 3,1 & $0,062^{* *}$ \\
\hline Hipotireoidismo & 3,9 & 0,0 & 2,7 & $0,315^{* *}$ \\
\hline Asma & 2,0 & 0,0 & 1,4 & $1,000^{* *}$ \\
\hline $\begin{array}{l}\text { Doença pulmonar } \\
\text { obstrutiva crônica }\end{array}$ & 1,0 & 1,1 & 1,0 & $0,227^{* *}$ \\
\hline Glaucoma & 0,5 & 2,2 & 1,0 & $0,555^{* *}$ \\
\hline Insuficiência venosa & 1,5 & 0,0 & 1,0 & \\
\hline
\end{tabular}

Notas: *Teste do qui-quadrado; ${ }^{* *}$ teste exato de Fisher.

\section{Incidência de comorbidades (\%)}

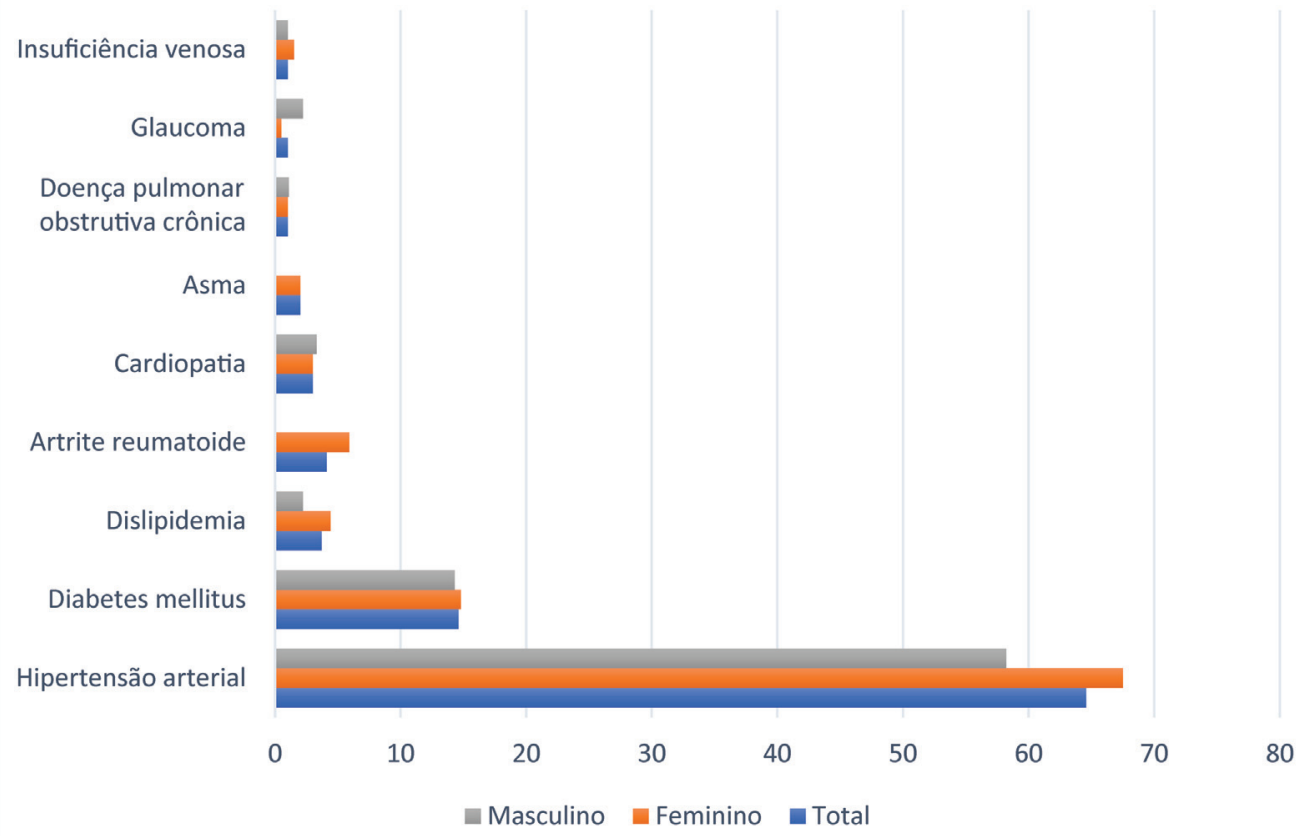

Fig. 1 Distribuição das patologias mais comuns na amostra total e por sexo.

das indicações mais restritas e do diferente perfil populacional, o déficit de assistência e problemas com a notificação contribuem para essa diferença tão grande. Há previsão de um aumento exponencial, impulsionado por mudanças populacionais e maior acesso aos serviços de saúde, sendo importante direcionar os recursos para os pacientes que obterão maiores benefícios com o procedimento. ${ }^{13}$

A amostra foi composta por um número maior de pacientes do sexo feminino, o que reflete a incidência da $O A$, que atinge de duas a três mulheres para cada homem. ${ }^{14}$ Embora seja controverso na literatura, o índice de satisfação após ATJ não parece divergir entre homens e mulheres. Singh et al., ${ }^{15}$ após avaliarem quase 18 mil pacientes submetidos a ATJ, encontraram nos homens a maior chance de infecção (+31\%), de readmissão nos primeiros 30 dias $(+25 \%)$, de revisão nos primeiros 5 anos ( $+20 \%$ ), e o maior índice de mortalidade no primeiro ano (+48\%). Robinson et al. ${ }^{16}$ estudaram 32.848 pacientes submetidos a ATJ, e concluíram que o sexo feminino é um fator protetor para sepse e para complicações cardiovasculares e renais, embora apresente maior risco de 
Tabela 4 Frequência das comorbidades clínicas por faixa etária

\begin{tabular}{|l|l|l|l|l|l|}
\hline Faixa etária/Comorbidades & $41-50$ anos & $51-60$ anos & $61-70$ anos & $71-80$ anos & $>80$ anos \\
\hline Número & 3 & 36 & 128 & 113 & 14 \\
\hline Hipertensão arterial sistêmica & $33,3 \%$ & $56,6 \%$ & $60,6 \%$ & $69,9 \%$ & $64,3 \%$ \\
\hline Diabetes mellitus & - & $16,7 \%$ & $16,5 \%$ & $20,4 \%$ & $7,1 \%$ \\
\hline Artrite reumatoide & $33,3 \%$ & $16,7 \%$ & $0,8 \%$ & $1,8 \%$ & $7,1 \%$ \\
\hline Dislipidemia & - & $2,8 \%$ & $3,2 \%$ & $4,4 \%$ & - \\
\hline Cardiopatia & - & $2,8 \%$ & $3,1 \%$ & $3,5 \%$ & - \\
\hline Hipotireoidismo & - & $2,8 \%$ & $3,1 \%$ & $0,9 \%$ & $7,1 \%$ \\
\hline Asma & - & 0 & $0,8 \%$ & $1,8 \%$ & - \\
\hline Doença pulmonar obstrutiva crônica & - & $2,8 \%$ & $0,8 \%$ & $0,9 \%$ & - \\
\hline Glaucoma & - & - & - & $2,7 \%$ & - \\
\hline Insuficiência venosa & - & - & $0,8 \%$ & $1,8 \%$ & - \\
\hline $\begin{array}{l}\text { Média do índice funcional de } \\
\text { comorbidades funcionais }\end{array}$ & $1,7 \pm 0,6$ & $1,8 \pm 0,6$ & $1,8 \pm 0,8$ & $1,8 \pm 0,8$ & $1,6 \pm 0,5$ \\
\hline $\begin{array}{l}\text { Mediana do índice funcional de } \\
\text { comorbidades funcionais }\end{array}$ & 2,0 & 2,0 & 2,0 & 2,0 & 2,0 \\
\hline $\begin{array}{l}\text { Média do índice modificado de } \\
\text { cinco fatores de fragilidade }\end{array}$ & $0,07 \pm 0,12$ & $0,16 \pm 0,15$ & $0,16 \pm 0,14$ & $0,19 \pm 0,13$ & $0,14 \pm 0,12$ \\
\hline $\begin{array}{l}\text { Mediana do índice modificado } \\
\text { de cinco fatores de fragilidade }\end{array}$ & 0,00 & 0,20 & 0,20 & 0,20 & 0,20 \\
\hline
\end{tabular}

desenvolver infecção urinária, de precisar de transfusão sanguínea, e de ter dificuldade de carga no membro operado.

Para a avaliação dos resultados cirúrgicos, é importante conhecer o perfil epidemiológico dos pacientes tratados, pois diversas comorbidades clínicas podem impactar diretamente o desfecho, ${ }^{17}$ comprometendo a demanda metabólica, a cicatrização da ferida operatória, e a administração da anestesia. ${ }^{18}$ Apesar dos benefícios associados à ATJ, até $24 \%$ dos pacientes operados podem enfrentar sérios eventos adversos, sendo primordial considerar quem deve ser submetido ao procedimento. A avaliação pré-operatória visa otimizar clinicamente o paciente, minimizando seus riscos e facilitando sua recuperação. ${ }^{19}$

Utilizamos o diagnóstico autorrelatado como ferramenta para listar as comorbidades, por se tratar de um método atrativo para doenças crônicas. Najafi et al. ${ }^{20}$ afirmaram que essa estratégia é confiável e eficiente, e apresenta algumas vantagens com relação à análise do prontuário, pois não depende do viés do notificador. Além disso, por motivos administrativos e contratuais, essas patologias podem estar omitidas e subnotificadas. Todos os pacientes da nossa amostra foram tratados pelo Sistema Único de Saúde (SUS), o que é ideal para este tipo de estudo, pois eles não sofreram pressão da seguradora, e não houve influência para a omissão de patologias.

A comorbidade mais frequentemente encontrada foi a hipertensão arterial sistêmica (HAS), que atingiu $64,9 \%$ da amostra. A maior incidência ocorreu na oitava década de vida (69,9\%). A prevalência da doença foi semelhante nos grupos masculino e feminino $(p=0,125)$. Esse resultado é condizente com o descrito por Feng et al.,21 que destacam a HAS como a comorbidade mais comum na prática ortopédica. A literatura é escassa em estudos sobre a possível influência da HAS no resultado final de pacientes submetidos à ATJ. Elmallah et al. ${ }^{22}$ encontraram resultados funcionais piores em hipertensos quando comparados a normotensos, embora a melhora da dor tenha sido semelhante. Dessa forma, a HAS pode influenciar negativamente 0 resultado funcional da cirurgia. A dislipidemia atingiu menos de $5 \%$ dos pacientes, sendo também mais frequente na oitava década de vida. $O$ uso de estatinas deve ser continuado, pois existem evidências de que essa medicação pode diminuir tanto o índice de mortalidade precoce quanto a chance de soltura asséptica da prótese nos primeiros dez anos. ${ }^{19}$

O diabetes mellitus (DM) esteve presente em 14,6\% da amostra total, sendo maior na oitava década de vida $(20,4 \%)$. Não houve diferença entre os sexos $(p=0,912)$. Segundo Flor e Campos, ${ }^{23}$ a incidência na população brasileira acima de 65 anos é de $16,7 \%$. A amostra incluiu pacientes abaixo dessa faixa etária, entre 41 e 50 anos, que não apresentaram DM e influenciaram a média total. O DM é uma patologia que interfere na cicatrização das feridas, diminui a atividade osteoblástica, e prejudica o sistema imunológico. ${ }^{19}$ Pacientes diabéticos têm um aumento no índice de complicações, como infecção periprotética (risco relativo - RR: 1,6), trombose venosa profunda (RR: 2,57), e soltura asséptica (RR: 9,36). ${ }^{24}$ Singh e Lewallen ${ }^{25}$ avaliaram 7.139 pacientes diabéticos submetidos a ATJ, e concluíram que o DM é um fator de risco independente para resultados desfavoráveis após a cirurgia. Apesar do maior número de complicações e resultados funcionais piores, os pacientes diabéticos apresentam um índice de satisfação semelhante ao dos não diabéticos, e o 
procedimento é capaz de gerar outros benefícios, como a redução do IMC em 50\% dos operados. ${ }^{26}$ Atenção deve ser dada aos níveis glicêmicos pré-operatórios, sendo o valor de $200 \mathrm{mg} / \mathrm{dL}$ o limite máximo. ${ }^{27}$

A análise da idade revelou que $82 \%$ dos pacientes encontram-se entre a sétima e oitava décadas de vida. Esses dados refletem as indicações mais restritas dessa cirurgia em pacientes latinos. Embora pacientes mais jovens tenham uma recuperação mais rápida e ótimos resultados funcionais, ${ }^{28}$ estes apresentam menor índice de satisfação e maior risco de revisão. A expectativa de vida no Brasil tem aumentado exponencialmente, e alcançou 76,74 anos para nascidos em 2020. Projeções indicam que, em 2050, ela alcançará 81,29 anos. Cher et al. ${ }^{29}$ avaliaram 209 octogenários submetidos a ATJ e encontraram excelentes resultados funcionais, que não diferiram dos de seus pares mais jovens. Porém, os autores destacam que, aomemso tempo que há melhora na qualidade de vida, há um aumento do custo para se oferecer o tratamento cirúrgico a pacientes cada vez mais idosos. Cada ano acrescentado à idade aumenta a chance de reinternação e de mortalidade. ${ }^{30}$

A obesidade é um fator claramente envolvido na gênese e na progressão da OA do joelho. ${ }^{6}$ Esse fato foi comprovado na nossa amostra, em que apenas $16,9 \%$ dos pacientes foram normotróficos, sendo o restante com sobrepeso ou obesos. O sexo feminino esteve associado ao maior IMC $(p=0,017)$. A obesidade é um fator de risco independente para complicações per e pós-operatórias, como infarto agudo do miocárdio, acidente vascular cerebral, infecção periprotética, e necessidade de revisão, ${ }^{19,21}$ além da maior chance de reinternação e mortalidade. ${ }^{30}$ Várias instituições têm restringido a realização de ATJ em pacientes com IMC maior do que $40 \mathrm{~kg} / \mathrm{m}^{2}$, devido ao maior custo e chance de eventos desfavoráveis. ${ }^{21} \mathrm{O}$ controle ponderal deve ser encorajado para pacientes com IMC acima de 25 $\mathrm{kg} / \mathrm{m}^{2}$, e a cirurgia bariátrica pode ser um recurso anterior à ATJ para obesos reistentes ao tratamento conservador. Traven et al. ${ }^{11}$ criaram um protocolo com cinco fatores (mFI-5) capazes de antever a chance de complicação após ATJ. Na amostra estudada, a obesidade foi o único fator diretamente relacionado ao mFI-5 ( $p=0,022)$, o que comprovou a relação do excesso de peso corporal com um prognóstico pior.

Apesar do sucesso da ATJ em restaurar a função e melhorar a qualidade de vida, até $20 \%$ dos pacientes podem apresentar dor ou insatisfação no pós-operatório. Vários autores desenvolveram ferramentas a fim de predizer o resultado funcional. Groll et al. ${ }^{10}$ desenvolveram o FCI. Diferente de outros índices, que avaliam o tempo de internação e o índice de mortalidade, o $\mathrm{FCI}$ indica o provável resultado funcional dos pacientes, com $77 \%$ de eficácia. Os autores incluíram 18 patologias no questionário, que pode ser autoaplicável. As perguntas incluem a avaliação da saúde mental e doenças respiratórias, fatores importantes ligados ao resultado da ATJ. A amostra estudada apresentou um prognóstico funcional pior para os obesos $(p<0,001)$ e pacientes do sexo feminino $(p=0,038)$, o que é condizente com os achados de Robinson et al., ${ }^{16}$ que descreveram maior dificuldade de deambulação após a cirurgia em pacientes femininos e obesos.

Este estudo apresenta algumas limitações. Por ser um estudo observacional e transversal, os pacientes não tiveram seu desfecho analisado. Porém, acreditamos que os resultados cumprem o objetivo de definir o perfil epidemiológico dos pacientes submetidos à ATJ. Os instrumentos incluídos, FCI e mFI-5, são ferramentas interessantes, mas não são capazes de estabelecer um limite para a realização da ATJ. Portanto, a participação dos pacientes e familiares no processo decisório deve ser estimulada.

\section{Conclusão}

Os pacientes submetidos à ATJ são essencialmente portadores de comorbidades clínicas que podem influenciar negativamente os resultados funcionais e elevar o índice de complicação da cirurgia. A identificação dos fatores de risco contribui para a segurança e melhor seleção dos candidatos.

\section{Suporte Financeiro}

Não houve suporte financeiro de fontes públicas, comerciais, ou sem fins lucrativos.

Conflito de Interesses

Os autores declaram não haver conflito de interesses.

\section{Referências}

1 da Silva RR, Santos AA, de Sampaio Carvalho Júnior J, Matos MA. Quality of life after total knee arthroplasty: systematic review. Rev Bras Ortop 2014;49(05):520-527

2 Zanasi S. Innovations in total knee replacement: new trends in operative treatment and changes in peri-operative management. Eur Orthop Traumatol 2011;2(1-2):21-31

3 Schwechter EM, Fitz W. Design rationale for customized TKA: a new idea or revisiting the past? Curr Rev Musculoskelet Med 2012;5(04):303-308

4 Ministério da Saúde. Secretaria Executiva. Datasus [acesso em: 28 de março de 2020]. Informações de Saúde. Disponível em: http:// tabnet.datasus.gov.br/cgi/tabcgi.exe?sih/cnv/qiuf.def

5 Agência Nacional de Saúde Suplementar ANS [acesso em 28 de Março de 2020]. Painel de dados do TISS 2018. Disponível em: https://app.powerbi.com/view? $\mathrm{r}=$ eyJrIjoiMDNIMDNINzEtZDA5Yy00YzE5LTg2Y2ItY2IxNTBkMTIwM2Q5IiwidCI6IjlkYmE0ODBjLTRmYTctNDJmNC1iYmEzLTBmYjEzNzVmYmU1Zij9

6 Loures FB, de Araújo Góes RF, Labronici PJ, Barretto JM, Olej B. Evaluation of body mass index as a prognostic factor in osteoarthrosis of the knee. Rev Bras Ortop 2016;51(04):400-404

7 Ferreira MC, Oliveira JCP, Zidan FF, Franciozi CES, Luzo MVM, Abdalla RJ. Artroplastia total de joelho e quadril: a preocupante realidade assistencial do Sistema Único de Saúde brasileiro. Rev Bras Ortop 2018;53(04):432-440

8 Bjorgul K, Novicoff WM, Saleh KJ. Evaluating comorbidities in total hip and knee arthroplasty: available instruments. J Orthop Traumatol 2010;11(04):203-209

9 Healy WL, Della Valle CJ, Iorio R, et al. Complications of total knee arthroplasty: standardized list and definitions of the Knee Society. Clin Orthop Relat Res 2013;471(01):215-220

10 Groll DL, To T, Bombardier C, Wright JG. The development of a comorbidity index with physical function as the outcome. J Clin Epidemiol 2005;58(06):595-602

11 Traven SA, Reeves RA, Sekar MG, Slone HS, Walton ZJ. New 5Factor Modified Frailty Index Predicts Morbidity and Mortality in Primary Hip and Knee Arthroplasty. J Arthroplasty 2019;34(01): 140-144 
12 Keyes GW, Carr AJ, Miller RK, Goodfellow JW. The radiographic classification of medial gonarthrosis. Correlation with operation methods in 200 knees. Acta Orthop Scand 1992;63(05):497-501

13 Dakin H, Gray A, Fitzpatrick R, Maclennan G, Murray DKAT Trial Group. Rationing of total knee replacement: a cost-effectiveness analysis on a large trial data set. BMJ Open 2012;2(01):e000332

14 Ro DH, Lee DY, Moon G, et al. Sex differences in knee joint loading: Cross-sectional study in geriatric population. J Orthop Res 2017; 35(06):1283-1289

15 Singh JA, Kwoh CK, Richardson D, Chen W, Ibrahim SA. Sex and surgical outcomes and mortality after primary total knee arthroplasty: a risk-adjusted analysis. Arthritis Care Res (Hoboken) 2013;65(07):1095-1102

16 Robinson J, Shin JI, Dowdell JE, Moucha CS, Chen DD. Impact of Gender on 30-Day Complications After Primary Total Joint Arthroplasty. J Arthroplasty 2017;32(08):2370-2374

17 Santos AC, Biagi AC. Epidemiological profile of patients undergoing knee arthroplasty from the physiotherapy service of a public hospital in Santo André-SP. ABCS Health Sci 2013;38 (01):2-7

18 Skou ST, Roos EM, Laursen MB, et al. A Randomized, Controlled Trial of Total Knee Replacement. N Engl J Med 2015;373(17): 1597-1606

19 Adie S, Harris I, Chuan A, Lewis P, Naylor JM. Selecting and optimising patients for total knee arthroplasty. Med J Aust 2019;210(03):135-141

20 Najafi F, Moradinazar M, Hamzeh B, Rezaeian S. The reliability of selfreporting chronic diseases: how reliable is the result of populationbased cohort studies. J Prev Med Hyg 2019;60(04):E349-E353

21 Feng JE, Novikov D, Anoushiravani AA, Schwarzkopf R. Total knee arthroplasty: improving outcomes with a multidisciplinary approach. J Multidiscip Healthc 2018;11:63-73
22 Elmallah RD, Cherian JJ, Robinson K, Harwin SF, Mont MA. The Effect of Comorbidities on Outcomes following Total Knee Arthroplasty. J Knee Surg 2015;28(05):411-416

23 Flor LS, Campos MR. Prevalência de diabetes mellitus e fatores associados na população adulta brasileira: evidências de um inquérito de base populacional. Rev Bras Epidemiol 2017;20 (01):16-29

24 Yang Z, Liu H, Xie X, Tan Z, Qin T, Kang P. The influence of diabetes mellitus on the post-operative outcome of elective primary total knee replacement: a systematic review and meta-analysis. Bone Joint J 2014;96-B(12):1637-1643

25 Singh JA, Lewallen DG. Diabetes: a risk factor for poor functional outcome after total knee arthroplasty. PLoS One 2013;8(11): e78991

26 Teo BJX, Chong HC, Yeo W, Tan AHC. The Impact of Diabetes on Patient Outcomes After Total Knee Arthroplasty in an Asian Population. J Arthroplasty 2018;33(10):3186-3189

27 Parvizi J, Shohat N, Gehrke T. Prevention of periprosthetic joint infection: new guidelines. Bone Joint J 2017;99-B(4, Supple B) 3-10

28 Kleeblad LJ, van der List JP, Zuiderbaan HA, Pearle AD. Larger range of motion and increased return to activity, but higher revision rates following unicompartmental versus total knee arthroplasty in patients under 65: a systematic review. Knee Surg Sports Traumatol Arthrosc 2018;26(06):1811-1822

29 Cher EWL, Tay KS, Zhang K, Tan SB, Howe TS, Koh JSB. The Effect of Comorbidities and Age on Functional Outcomes After Total Knee Arthroplasty in the Octogenarian: A Matched Cohort Study. Geriatr Orthop Surg Rehabil 2018;9:2151459318769508

30 Lehtonen EJ, Hess MC, McGwin G Jr, Shah A, Godoy-Santos AL, Naranje S. Risk factors for early hospital readmission following total knee arthroplasty. Acta Ortop Bras 2018;26(05):309-313 